\title{
Bingimering Jourtilal
}

Article

\section{Propagation of Own Non- Axisymmetric Waves in Viscoelastic Three-Layered Cylindrical Shells}

\author{
I. I. Safarov ${ }^{1,3, a}$, M. Kh. Teshaev ${ }^{2,3,4, b, *}$, A. M. Marasulov ${ }^{5, c}$, and B. Z. Nuriddinov ${ }^{1, d}$ \\ 1 Tashkent Chemical-Technological Institute, 32, Navoi Str., Tashkent, Republic of Uzbekistan \\ 2 Institute of Mathematics AS R Uz, Bukhara Branch, 11, M. Iqbol Str., Bukhara, Republic of Uzbekistan \\ 3 Bukhara Engineering-Technological Institute, 15, K. Murtazayev Str., Bukhara, Republic of Uzbekistan \\ 4 Tashkent Institute of Irrigation and Mechanization Engineering in Agricultural, Bukhara Branch, 32, Gazli \\ Highway, Bukhara, Bukhara, Republic of Uzbekistan \\ 5 International Kazakh-Turkish University, 29, B. Sattarkhanov Ave., Turkestan Sity, Turkestan Region, \\ Kazakhstan \\ E-mail: asafarov54@mail.ru,b,*muhsin_5@mail.ru (Corresponding author),cMarasulov11@mail.ru, \\ dB.Nuriddinov1985@mail.ru
}

\begin{abstract}
The relevance of the study of the dynamic movements of structures consisting of a thin-walled shell and a viscoelastic cylindrical cavity mounted on it is due to their widespread application in modern technology. The mechanical system under consideration consists of two concentric cylindrical shells with a viscoelastic filler (or cylinder) between the shells. The filler and shell can be firmly attached to the outer and inner shells along the entire cylindrical surface. The basic equations of small oscillations of the shell theory and the three-dimensional viscoelasticity theory are used to describe the oscillations of the "shell-filler-shell" system with the exact satisfaction of the contact boundary. The main purpose of the work is to develop a method and algorithm for calculating the problems of propagation and absorption of natural waves in a mechanical "shell-filler-shell" mechanical system. A calculation method based on Müller, Gauss and orthogonal running methods was developed. The Kirchhoff-Love and Tymoshenko hypotheses are used for the cylindrical shell. For dissipative homogeneous and non-homogeneous mechanical systems, the variation of the real and imaginary parts of the complex phase velocity from different system parameters was studied. For sufficiently long waves, Kirchhoff-Love and Tymoshenko hypothesized that the phase velocities of the first form were found to be well matched. It was also found that it is possible to use shell equations for shortwave, taking into account the compression of the filler. It was found that the increase in filler thickness was particularly significant for the relatively small thickness of the filler.
\end{abstract}

Keywords: Non-axisymmetric waves, three-layer cylindrical shell, phase velocity, wave number, filler.

ENGINEERING JOURNAL Volume 25 Issue 7

Received 10 June 2021

Accepted 8 July 2021

Published 31 July 2021

Online at https:// engj.org/

DOI:10.4186/ej.2021.25.7.97

This article is based on the presentation at The Construction Digitalization for Sustainable Development (CDSD 2020) International Conference in Hanoi, Vietnam, 24th-25th November 2020. 


\section{Introduction}

One of the main directions in the development of modern industrial production is the widespread use of resource-saving design solutions and technologies that are directly related to the reduction of material consumption of these structures. To do this, it is necessary to study the dissipative properties of structural materials in general. Such structures are widely used in aircraft and missile construction $[1,2,3]$. This is primarily due to the fact that three-layer structures consisting of thin iscoelastic load-bearing layers and a deformable aggregate (polymeric material) have less weight with equal rigidity compared to the same structures with arbitrary thickness. The middle floor can also serve as a vibration insulator of the object. In addition, the middle layer can play the role of thermal insulator of the structure [4].

In [5-8], the dynamic and static states of three-layer cylindrical structures with a filler are considered. Gas, water, and other elastic materials can be used as a filler. It is noted that the consideration of the inhomogeneity of the filler is not sufficiently studied. In particular, it is very important for practical calculations to study the dynamic state of three-layer structures, taking into account structural heterogeneity and viscoelasticity under vibration effects. A significant number of works are devoted to solving this problem [9-11].

However, until now, no general methods have been developed for calculating structurally inhomogeneous layered cylindrical shells surrounded or filled with a linear continuous medium. A sufficiently detailed analysis of the state of the problem of vibrations of shells interacting with a liquid medium is contained in $[12,13$, 14], and for shells with an elastic filler - in [15]. The problems of oscillations in a system of shells with a filler are considered in $[16,17]$.

In [18], the problem of propagation of eigenwaves in three-layer plates is investigated. The problem is posed in a refined formulation of the theory of plates and shells. The equations of the oscillatory processes of the filler are described by the Lame equations and for shells the shell equations obeying the Kirchhoff -Love hypotheses are used. The dispersion relations are constructed in the form of algebraic equations. The algebraic equations are solved numerically and the phase velocities for symmetric and antisymmetric waves are determined [19].

Existing mathematical formulations of the problem and methods for calculating three-layer structures do not take into account the viscoelastic or rheological properties of materials.

Thus, the development of methods for the dynamic calculation of three-layer shells based on dissipativeinhomogeneous mathematical models and their implementation in the form of computational algorithms represent actual problems of mechanics. In contrast to other works, this paper studies the propagation of nonaxisymmetric eigenwaves in viscoelastic three-layer cylindrical bodies when rigid (or sliding) contact conditions are met at the interface of the layers. A dispersion equation with a complex output parameter is obtained. The real and imaginary parts of the complex phase velocity as a function of the wave numbers and other parameters of the mechanical system are investigated. The behavior of the energy dissipation coefficient on the geometrical and physico-mechanical parameters of the structure is found.

\section{Methods}

\subsection{Problem Statement and Basic Relations}

Consider the propagation of eigenwaves in infinitely long structures consisting of two outer shells, between which there is a viscoelastic filler. Equations of vibration of a viscoelastic cylindrical aggregate, in the coordinate system $r, \theta, z$ written as [20].

$$
\tilde{\mu}_{s} \nabla^{2} \vec{u}+\left(\tilde{\lambda}_{s}+\tilde{\mu}_{s}\right) \text { graddiv } \vec{u}=\rho_{s} \frac{\partial^{2} \vec{u}}{\partial t^{2}}
$$

where $\vec{u}\left(u_{r}, u_{\theta}, u_{z}\right)$ - vector of displacement of points of the environment; $\rho_{s^{-}}$- density of the filler material:

$$
\begin{gathered}
\operatorname{grad} \varphi=\frac{\partial \varphi}{\partial r} \vec{k}_{r}+\frac{1}{r} \frac{\partial \varphi}{\partial \theta} \vec{k}_{\theta}+\frac{\partial \varphi}{\partial z} \vec{k}_{z} ; \\
\operatorname{div} \vec{u}=\frac{1}{r} \frac{\partial u_{\theta}}{\partial \theta}+\frac{1}{r} \frac{\partial\left[r u_{r}\right]}{\partial r}+\frac{\partial u_{z}}{\partial z} ; \\
\nabla^{2} \vec{u}=\frac{1}{r} \frac{\partial}{\partial r}\left(r \frac{\partial \vec{u}}{\partial r}\right)+\frac{1}{r^{2}} \frac{\partial^{2} \vec{u}}{\partial \theta^{2}}+\frac{\partial^{2} \vec{u}}{\partial z^{2}} ; \\
\tilde{\lambda}_{s} \varphi(t)=\lambda_{0 s}\left[\varphi(t)-\int_{0}^{t} R_{\lambda s}(t-\tau) \varphi(\tau) d \tau\right] ; \\
\tilde{\mu}_{s} \varphi(t)=\mu_{0 s}\left[\varphi(t)-\int_{0}^{t} R_{\mu s}(t-\tau) \varphi(\tau) d \tau\right],
\end{gathered}
$$

$R_{\lambda S}(t-\tau)$ and $R_{\mu S}(t-\tau)$ - relaxation cores; $\lambda_{0 s}, \mu_{0 s^{-}}$instantaneous elastic modulus; $\varphi(t)$ - arbitrary time function; $\vec{k}_{r}, \vec{k}_{\theta}, \vec{k}_{z}$ - unit vectors by direction $r, \theta, z$.

The equations of motion of three-layer shells, which are usually used in calculations, are obtained using various assumptions related to the nature of the filler deformation [21].

In order to assess the acceptability of the results obtained on the basis of these equations, when solving dynamic problems for three - layer cylindrical shells, a refined approach is proposed, when the motion of the filler is described by the dynamic equations of the theory of viscoelasticity (1), and the bearing layers are considered as thin shells, obeying the hypotheses of Kirchhoff-Love or Timoshenko. The contact between the carrier layers and the aggregate can be rigid or sliding. 
Integro-differential equations of vibrations of shells (carrier layers) in vector and matrix form take the following form:

$$
\begin{gathered}
L_{i j} \vec{U}_{k}-\int_{0}^{t} L_{i j} R_{E \kappa}(t-\tau) \vec{U}_{k}(\vec{r}, \tau) d \tau \\
=\frac{\left(1-v_{0 \kappa}{ }^{2}\right)}{G_{0 \kappa} h_{0 \kappa}} \vec{q}_{\kappa} \\
+\rho_{0 \kappa} \frac{\left(1-v_{0 \kappa}{ }^{2}\right)}{G_{0 \kappa}} \frac{\partial^{2} \vec{U}_{k}}{\partial t^{2}} . \\
(k=1,2)
\end{gathered}
$$

Here $v_{0 \kappa}$ - the Poisson's ratio of the shell, is considered a constant value $U_{k}\left(u_{k}, \vartheta_{k}, w_{k}\right)$ - vector of displacement of points of the median surface of the shell; index $k=1$ refers to the inner shell, and $k=2$ - to the outer shell, $\rho_{0 \kappa}$-is density of the carrier layer material, $\vec{q}_{\mathrm{K}}\left(q_{k r}, q_{k \theta}, q_{k z}\right)$ - external loads that are applied from the placeholder side or a specified external load; $R_{E k}(t-$ $\tau)$ - the relaxation core of the $k^{\text {th }}$ carrier layer. Equations the movements of the cylindrical shell obtained on the basis of the Kirchhoff-Love hypotheses take the following form

$$
\begin{aligned}
& L_{11}=\frac{\partial^{2}}{\partial z^{2}}+\frac{1-v_{k}}{2 R_{k}^{2}} \frac{\partial^{2}}{\partial \theta^{2}}-\rho_{k} \frac{1-v_{k}}{2 G_{k 0}} \frac{\partial^{2}}{\partial t^{2}} \\
& L_{12}=L_{21}=\frac{1+v_{k}}{2 R_{k}} \frac{\partial^{2}}{\partial z \partial \theta} \\
& L_{13}=L_{31}==\frac{v_{k}}{R_{k}} \frac{\partial}{\partial z} \\
& L_{22}=\frac{1-v_{k}}{2 R_{k}^{2}} \frac{\partial^{2}}{\partial z^{2}}+\frac{1}{R_{k}^{2}} \frac{\partial^{2}}{\partial \theta^{2}}-\rho_{k} \frac{1-v_{k}}{2 G_{k 0}} \frac{\partial^{2}}{\partial t^{2}} \\
& L_{33}=\frac{h_{k}^{2}}{12} \nabla^{2} \nabla^{2}+\frac{1}{a_{k}^{2}}+\rho_{k} \frac{1-v_{k}}{2 G_{k 0}} \frac{\partial^{2}}{\partial t^{2}} \\
& \nabla^{2} \nabla^{2}=\frac{\partial^{4}}{\partial z^{4}}+\frac{2}{a_{k}^{2}} \frac{\partial^{4}}{\partial z^{2} \partial \theta^{2}}+\frac{1}{a_{k}^{4}} \frac{\partial^{4}}{\partial \theta^{4}}
\end{aligned}
$$

Here $R_{k}$-is the radius of the middle surface, $h_{k}$-is the thickness, $G_{k 0}$-is the instant modulus of elasticity.

Load vector's components:

$$
\begin{aligned}
\left\{q_{k r}, q_{k \theta}, q_{k z}\right\}= & -\frac{1-v_{k}}{2 G_{k 0} h_{k}}\left\{p_{r k}+q_{r k}, p_{\theta k}\right. \\
& \left.+q_{\theta k}, p_{z k}+q_{z k}\right\}
\end{aligned}
$$

Here $q_{r k}, q_{\theta k}, q_{z k^{-}}$radius of the middle surface of the carrier layer; $p_{r k}, p_{\theta k}, p_{z k^{-}}$the intensity of the external specified load in the corresponding direction. At the contact of the shells with the filler, the conditions of sliding contact are met. When using the Kirchhoff-Love hypotheses, these boundary conditions will take the form:

$$
\begin{gathered}
r=R_{k}: \quad u_{r}=w_{k} ; \sigma_{r z}=\sigma_{r \theta}=0 ; \\
r=R_{1}: \sigma_{r r}=-q_{r 1} ; r=R_{1}: \sigma_{r r}=-q_{r 2} .
\end{gathered}
$$

It is assumed that the contact of the shells (bearing layers) with the deformable aggregate occurs along the median surfaces of the layers. If the hard contact conditions are met at the boundary of the shells with the filler, then the following equalities are assumed:

$$
\begin{gathered}
u_{r}=w_{k} ; u_{\theta}=\vartheta_{k} ; u_{z}=u_{k} ; \\
\sigma_{r r}=\mp q_{r k} ; \sigma_{r \theta}=\mp q_{\theta k} ; \sigma_{r z}=\mp q_{z k} ;
\end{gathered}
$$

The minus and plus signs correspond to $k=1$ and $k=2$, accordingly. If we use the hypotheses of Timoshenko, then we get the same five equations. In the study of the natural oscillations of the mechanical system under consideration, the components of a given load $p_{r k}, p_{\theta k}, p_{z k}$ accepted as zero.

It is assumed that the integral terms in expressions (2) and (3) are sufficiently small. In this case, the amplitudes of the ratio $\phi(t)=\psi(t) e^{-i \omega_{R} t}$, a slowly changing function of time, $\omega_{R}$-the actual value. The assumption made will allow us to apply the freezing procedure [22, 23] and, then the relations (2) are replaced by the following approximate relations

$$
\begin{aligned}
& \bar{\lambda}_{s}[\varphi(t)]=\lambda_{0 s}\left[1-\Gamma_{s \lambda}^{C}\left(\omega_{R}\right)-i \Gamma_{s \lambda}^{S}\left(\omega_{R}\right)\right] \\
& \bar{\mu}_{s}[\varphi(t)]=\mu_{0 s}\left[1-\Gamma_{s \mu}^{C}\left(\omega_{R}\right)-i \Gamma_{s \mu}^{S}\left(\omega_{R}\right)\right] ; \\
& \bar{G}_{k}[\varphi(t)]=G_{0 k}\left[1-\Gamma_{G_{k}}^{C}\left(\omega_{R}\right)-i \Gamma_{G_{k}}^{S}\left(\omega_{R}\right)\right],
\end{aligned}
$$

where

$$
\begin{gathered}
\Gamma_{s \lambda}{ }^{C}\left(\omega_{R}\right)=\int_{0}^{\infty} R_{s \lambda}(\tau) \cos \omega_{R} \tau d \tau, \Gamma_{s \mu}{ }^{C}\left(\omega_{R}\right) \\
=\int_{0}^{\infty} R_{s \mu}(\tau) \cos \omega_{R} \tau d \tau, \\
\Gamma_{s \lambda}{ }^{S}\left(\omega_{R}\right)=\int_{0}^{\infty} R_{s \lambda}(\tau) \sin \omega_{R} \tau d \tau, \Gamma_{s \mu}{ }^{S}\left(\omega_{R}\right) \\
=\int_{0}^{\infty} R_{s \mu}(\tau) \sin \omega_{R} \tau d \tau,
\end{gathered}
$$


The Rzhanitsin-Koltunov kernel $R_{s \lambda}(t)=R_{s \mu}(t)=$ $\frac{A e^{-\beta t}}{t^{-\alpha}}$ was used in the calculations. From Eq. (1) and (3) with substitution of expressions (8) and (9):

$$
\begin{gathered}
\bar{\mu}_{s} \nabla^{2} \vec{u}+\left(\bar{\lambda}_{s}+\bar{\mu}_{s}\right) \text { graddiv } \vec{u}=\rho_{s} \frac{\partial^{2} \vec{u}}{\partial t^{2}} \\
\bar{L}_{i_{i}}^{k} \vec{U}_{k}=\frac{\left(1-v_{0 \kappa}{ }^{2}\right)}{G_{0 \kappa} h_{0 \kappa}} \vec{q}_{\mathrm{K}}+\rho_{0 \kappa} \frac{\left(1-v_{0 \kappa}{ }^{2}\right)}{G_{0 \kappa}} \frac{\partial^{2} \vec{U}_{k}}{\partial t^{2}}
\end{gathered}
$$

Here is the differential operator $\bar{L}_{i}^{k}[\varphi(t)]=$ $L_{i j}^{k}\left(1-\left(R_{i j}^{k} \delta_{i j}\right)^{-1}\right),[\varphi(t)]-$ Kronecker symbols) and $R_{i j}^{k}\left(R_{11}^{k}=R_{22}^{k}=R_{33}^{k}=\bar{G}_{k}[\varphi(t)]\right) \quad-\quad$ a third-order diagonal matrix for the Kirchhoff-Love hypotheses. And also for the Timoshenko hypotheses, the order of the diagonal matrix is five. Thus, we obtain a system of differential equations (10), (11) with complex coefficients, which is solved under the boundary conditions (6) and (7). Next, we study the behavior of eigenvalues and waveforms (spectral problem) in viscoelastic layered cylindrical bodies at given values of wave numbers $[24$, 25].

\subsection{Non-axisymmetric Waves in a Three-Layer Cylindrical Shell}

The main purpose of the study is to estimate the wave effect of the number of waves in the circumferential direction on the phase velocities of wave propagation and the dissipative properties of a mechanical system. In the non-axisymmetric case, the longitudinal-transverse waves and the torsion waves are connected. Their propagation velocities are determined from a single general dispersion equation.

For the non-axisymmetric case, the solutions of Eq. (11) are sought in the form

$$
\begin{aligned}
& \left\{u_{k}, w_{k}, u_{z}, u_{r}\right\} \\
& =\sum_{\substack{\infty=0 \\
\infty}}^{\infty}\left\{u_{k, n}, w_{k, n}, U_{n z}(r), U_{n r}(r)\right\} \cos (n \theta) \exp i(\xi z \\
& -\omega t) ;
\end{aligned}
$$

$$
\begin{aligned}
& \left\{v_{k}, u_{\theta}\right\} \\
& =\sum_{n=0}^{\infty}\left\{v_{k, n}, U_{n \theta}(r)\right\} \sin (n \theta) \exp i(\xi z-\omega t)
\end{aligned}
$$

Here $u_{k, n}, \vartheta_{k, n}, w_{k, n}, U_{n z}(r), U_{n \theta}(r), U_{n r}(r)$ - the amplitudes of the displacements of the shell and the filler, respectively, $\xi=2 \pi / \lambda_{f}$ - wave number, $\omega=\omega_{R}+$ $i \omega_{I}=c_{f} \xi$ - complex frequency, $\lambda_{f}, c_{f}$ - length and complex phase velocity, $\mathrm{n}$ is the number of waves in the circumferential direction.

Substituting (12) into (1), we obtain the following system of algebraic equations with complex coefficients

$$
\begin{aligned}
& \left(\left(\xi^{2}+\frac{1-v_{0 k}}{2 a_{k}^{2}} n^{2}\right)-\rho_{k} \frac{1-v_{0 k}}{2 \bar{G}_{k}} \omega^{2}\right) u_{k . n} \\
& +i \xi \frac{\left(1+v_{0 k}\right) n}{2 a_{k}} \vartheta_{k . n}+i \xi \frac{v_{0 k}}{a_{k}} w_{k . n} \\
& =0 \text {; } \\
& i \xi \frac{\left(1+v_{0 k}\right) n}{2 a_{k}} u_{k . n} \\
& +\left[\frac{1-v_{0 k}}{2}\left(\xi^{2}-\rho_{k} \frac{1-v_{0 k}}{2 \bar{G}_{k}} \omega^{2}\right)\right. \\
& \left.+\frac{n^{2}}{a_{k}^{2}}\right] \vartheta_{k . n}+\frac{n^{2}}{a_{k}^{2}} w_{k . n}=0, \\
& i \xi \frac{v_{0 k}}{2 a_{k}} u_{k . n}+\frac{n}{a_{k}^{2}} \vartheta_{k . n} \\
& +\left[\frac{h_{k}^{2}}{12}\left(\xi^{2}+\frac{n^{2}}{a_{k}^{2}}\right)^{2}-\rho_{k} \frac{1-v_{0 k}}{2 \bar{G}_{k}} \omega^{2}+\frac{1}{a_{k}^{2}}\right] w_{k . n} \\
& ==-\frac{1-v_{0 k}}{2 \bar{G}_{k} h_{k}} q_{r k, n}^{0} \text {. }
\end{aligned}
$$

From (13), we express the amplitudes of the loads that are transmitted to the filler through the amplitudes of the displacements of the points of the median surfaces of the bearing layers:

$$
\begin{gathered}
q_{r k, n}^{0}=-\frac{2 \bar{G}_{k} k^{2}}{1-v_{0 k}} f_{k} \frac{w_{k, n}}{h_{k}} ; f_{k} \\
=e_{3}-v_{0 k} \eta^{2} \frac{e_{6}}{\epsilon_{k}^{2}}+\frac{n^{2}}{\epsilon_{k}^{4}} \frac{e_{5}}{e_{4}} ; \\
\epsilon_{k}=\left\{\begin{array}{c}
\frac{a_{1}}{a_{2}}=1-k_{1} k_{2}, \text { if } \kappa=1, k_{s}=h_{s} / h_{2} \\
1, \quad \text { if } k=2 . \\
e_{1}=\left(1-\frac{1-v_{0 k}}{3} c_{0}^{2}\right) \eta^{2}+\frac{1-v_{0 k}}{2} \frac{n^{2}}{\epsilon_{k}^{2}} \\
e_{2}=\frac{1-v_{0 k}}{2}\left(1-\frac{2}{3} c_{0}^{2}\right) \eta^{2}+\frac{n^{2}}{\epsilon_{k}^{2}} \\
e_{3}=\frac{k^{2}}{12}\left(\eta^{2}+\frac{n^{2}}{\epsilon_{k}^{2}}\right)+\frac{1}{\epsilon_{k}^{2}}-\frac{1-v_{0 k}}{3} c_{0}^{2} \eta^{2} ; \\
e_{4}=\frac{\left(1+v_{0 k}\right)^{2}}{12} \frac{n^{2} \eta^{2}}{\epsilon_{k}^{2}}-e_{2} ; \\
e_{5}=1-\frac{v_{0 k}\left(1+v_{0 k}\right) \eta^{2}}{2 e_{1}} ;
\end{array}\right.
\end{gathered}
$$




$$
\begin{gathered}
e_{6}=\frac{\left(1+v_{0 k}\right) e_{5}}{2 e_{1} e_{4}} \frac{n^{2}}{\epsilon_{k}^{2}}+\frac{v_{0 k}}{e_{1}}, \\
k=1,2
\end{gathered}
$$

$\eta=\xi h_{2} / k_{2}, k_{k}=h_{k} / a_{2}, \mathrm{c}_{0 k}=c_{f}\left(\frac{3 \rho_{k}}{2 G_{0 k}}\right), \mathrm{c}_{0}=\omega / \xi$

$k_{s^{-}}$filler thickness

All dimensionless parameters are entered here according to (14). Assuming in (13), we arrive at the problem for axisymmetric propagation of eigenwaves on a three-layer cylindrical body [26].

To integrate the equations of motion of the placeholder, we introduce potential functions $\left(\varphi_{n}, \psi_{n}, \chi_{n}\right)[27]$

$$
\begin{gathered}
U_{n}(r)=i \xi \varphi_{n}+\frac{d^{2} \psi_{n}}{d r^{2}}+\frac{1}{r} \frac{d \psi_{n}}{d r}-\frac{n^{2}}{r^{2}} \psi_{n} \\
V_{n}(r)=-\frac{n}{r} \varphi_{n}+i \xi \frac{n}{r} \psi_{n}-\frac{d \chi_{n}}{d r} \\
W_{n}(r)=\frac{d \psi_{n}}{d r}-i \xi \frac{d \psi_{n}}{d r}+\frac{n}{r} \chi_{n}
\end{gathered}
$$

Substituting (15), taking into account (11), in (7), we obtain for potential functions the Bessel equation

$$
\begin{gathered}
\frac{d^{2} \Delta_{n j}}{d r^{2}}+\frac{1}{r} \frac{d \Delta_{n j}}{d r}-\left[\frac{n^{2}}{r^{2}}+\left(1-\bar{M}_{p s j}^{2}\right) \xi^{2}\right] \Delta_{n j}=0, \\
(j=1,2,3)
\end{gathered}
$$

Here $\Delta_{n 1}=\varphi_{n}, \Delta_{n 2}=\psi_{n}, \Delta_{n 3}=\chi_{n}, M_{p s 1}^{2}=\bar{M}_{p 1}^{2} M_{p}^{2}$, $M_{p s 2}^{2}=\bar{M}_{s 2}^{2} M_{s}^{2}, M_{p s 3}^{2}=\bar{M}_{s 3}^{2} M_{s}^{2}, M_{p 1}^{2}=G_{I p}, \bar{M}_{s 2}^{2}=$ $G_{I S}, \bar{M}_{s 3}^{2}=G_{I s}, M_{p}=c_{f} / c_{s p}, M_{s}=c_{f} / c_{s S}, G_{I p}=1-$ $\Gamma_{G p}^{c}\left(\omega_{R}\right)-i \Gamma_{G p}^{S}\left(\omega_{R}\right), G_{I S}=1-\Gamma_{G S}^{c}\left(\omega_{R}\right)-i \Gamma_{G S}^{S}\left(\omega_{R}\right)$, $c_{s p}^{2}=\frac{2 G_{0 p}\left(1-v_{s}\right)}{\rho_{s}\left(1-2 v_{s}\right)}, c_{s s}^{2}=\frac{G_{0 p}}{\rho_{s}}, G_{0 p^{-}}$moments modulus of elasticity.

The general solutions of Eq. (16) have the form:

$$
\begin{gathered}
\Delta_{n j}(r, \xi)=A_{n j}(\xi) J_{n}\left(m_{j} \xi r\right) \\
+B_{n j}(\xi) N_{n}\left(m_{j} \xi r\right)
\end{gathered}
$$

Here $m_{j}=\sqrt{1-M_{p s j}^{2}}, A_{n j}(\xi), B_{n j}(\xi)-$ is constants.
Stress amplitudes of filler:

$$
\begin{gathered}
\left.\sigma_{r r, n}=\bar{\lambda}_{s}\left(\frac{d^{2} \varphi_{n}}{d r^{2}}+\frac{1}{r} \frac{d \varphi_{n}}{d r}-\frac{n^{2}}{r^{2}} \varphi_{n}-\xi^{2} \varphi_{n}\right)\right) \\
+2 \bar{\mu}_{s}\left(\frac{d^{2} \varphi_{n}}{d r^{2}}-i \xi \frac{d^{2} \psi_{n}}{d r^{2}}\right. \\
\left.+\frac{n}{r} \frac{d \psi_{n}}{d r}-\frac{n}{r^{2}} \chi_{n}\right) ; \\
\sigma_{r x, n}=\bar{\mu}_{s}\left(2 i \xi \frac{d \varphi_{n}}{d r}+\frac{d^{3} \varphi_{n}}{d r^{3}}+\frac{1}{r} \frac{d^{2} \psi_{n}}{d r^{2}}\right. \\
-\frac{n^{2}+1}{r^{2}} \frac{d \psi_{n}}{d r}+\frac{2 n^{2}}{r^{3}} \psi_{n} \\
\left.+\xi \frac{d \psi_{n}}{d r}+i \frac{n \xi}{r} \chi_{n}\right) ; \\
\sigma_{r \theta, n}=\bar{\mu}_{s}\left(\frac{2 n}{r}\left(\frac{\varphi_{n}}{r}-\frac{d \varphi_{n}}{d r}\right)+i \frac{2 n \xi}{r} \frac{d \psi_{n}}{d r}-\right. \\
\left.i \frac{2 n}{r^{2}} \psi_{n}+\frac{1}{r} \frac{d \chi_{n}}{d r}--\frac{n^{2}}{r^{2}} \chi_{n}-\frac{d^{2} \chi_{n}}{d r^{2}}\right) .
\end{gathered}
$$

Equation (17) substituting into (18):

$$
\operatorname{det}_{n}\left\|a_{i j}\right\|=0(i, j=1, \ldots, 6)
$$

where the elements of the determinant are calculated by the formulas:

$$
\begin{gathered}
a_{11}=m_{1} \eta s_{1}-\frac{n}{\epsilon_{1}} s_{2} ; \\
a_{12}=-\left(m_{1} \eta s_{4}+\frac{n}{\epsilon_{1}} s_{5}\right) ; \\
a_{13}=\left(m_{2} \eta s_{7}-\frac{n}{\epsilon_{1}} s_{8}\right) t_{1} ; \\
a_{14}=\left(m_{2} \eta s_{10}+\frac{n}{\epsilon_{1}} s_{11}\right) t_{1} ; \\
a_{15}=\frac{n s_{8}}{2 \epsilon_{1}} ; \\
a_{16}=-\frac{n s_{11}}{2 \epsilon_{1}} ; \\
a_{21}=m_{1} \eta-n s_{3} ; \\
a_{21}=0 ; \\
a_{22}=-m_{1} \eta-n s_{6} ; \\
a_{23}=\left(m_{2} \eta-n s_{9}\right) t_{1} ; \\
a_{24}=\left(m_{2} \eta+n s_{12}\right) t_{1} ; \\
a_{25}=\frac{n s_{9}}{2} ;
\end{gathered}
$$




$$
\begin{aligned}
& a_{26}=n\left[m_{2} \eta \in_{1} s_{1}-(n-1) s_{2}\right] \\
& a_{31}=0 \text {; } \\
& a_{32}=-n\left[m_{2} \eta \epsilon_{1} s_{4}-(n-1) s_{5}\right] \text {; } \\
& a_{33}=n\left[m_{2} \eta \in_{1} s_{7}-(n-1) s_{8}\right] ; \\
& a_{34}=n\left[m_{2} \eta \in_{1} s_{10}+(n-1) s_{11}\right] \text {; } \\
& a_{35}=m_{2} \eta \epsilon_{1} s_{7}+\left[n(n-1)+\frac{m_{2}^{2} \eta^{2} \in_{1}^{2}}{2}\right] s_{8} ; \\
& a_{36}=m_{2} \eta \epsilon_{1} s_{10}-\left[n(n-1)+\frac{m_{2}^{2} \eta^{2} \epsilon_{1}^{2}}{2}\right] s_{11} \text {; } \\
& a_{41}=0 \\
& a_{42}=-n\left[m_{2} \eta-(n-1) s_{6}\right] \text {; } \\
& a_{43}=n\left[m_{2} \eta-(n-1) s_{9}\right] \text {; } \\
& a_{44}=n\left[m_{2} \eta-(n-1) s_{12}\right] ; \\
& a_{45}=m_{2} \eta-\left[n(n-1)+\frac{m_{2}^{2} \eta^{2}}{2}\right] s_{9} ; \\
& a_{46}=m_{2} \eta-\left[n(n-1)+\frac{m_{2}^{2} \eta}{2}\right] s_{12} ; \\
& a_{51}=m_{1} \eta t_{5} s_{1}+\left(t_{1} \eta^{2}+t_{6}\right) s_{2} \text {; } \\
& a_{52}=-m_{2} \eta t_{5} s_{4}+\left(t_{1} \eta^{2}+t_{6}\right) s_{5} \\
& a_{53}=m_{2} \eta t_{5} s_{7}+\left(m_{2}^{2} \eta^{2}+t_{6}\right) s_{8} ; \\
& a_{54}=m_{2} \eta t_{5} s_{10}-\left(m_{s}^{2} \eta^{2}+t_{6}\right) s_{11} ; \\
& a_{55}=\frac{n}{\epsilon_{1}}\left[m_{2} \eta s_{7}-\left(\frac{n-1}{\epsilon_{1}}-t_{2} t_{3}\right) s_{8}\right] ; \\
& a_{56}=\frac{n}{\epsilon_{1}}\left[m_{2} \eta s_{10}+\left(\frac{n-1}{\epsilon_{1}}-t_{2} t_{3}\right) s_{11}\right] \text {; } \\
& a_{61}=m_{1} \eta t_{7}+\left(t_{1} \eta^{2}+t_{8}\right) s_{3} ; \\
& a_{62}=-m_{1} \eta t_{7}+\left(t_{1} \eta^{2}+t_{8}\right) s_{6} ; \\
& a_{63}=m_{2} \eta t_{7}+\left(m_{s}^{2} \eta^{2}+t_{8}\right) s_{9} ; \\
& a_{64}=m_{2} \eta t_{7}-\left(m_{s}^{2} \eta^{2}+t_{8}\right) s_{12} ; \\
& a_{65}=n\left[m_{2} \eta-\left(n-1+t_{2} t_{4}\right) s_{9}\right] \text {; } \\
& a_{66}=n\left[m_{2} \eta+\left(n-1+t_{2} t_{4}\right) s_{12}\right] \text {; }
\end{aligned}
$$

$$
\begin{gathered}
t_{1}=\frac{1+m_{s}^{2}}{2} \\
t_{2}=\frac{k \gamma}{1-v} \\
t_{3}=f_{1} \\
t_{4}=f_{2}
\end{gathered}
$$$$
t_{7}=1-t_{2} t_{4}
$$$$
t_{5}=\frac{1}{\epsilon_{1}}+t_{2} t_{3}
$$$$
t_{6}=\frac{n(n-1)}{\epsilon_{1}^{2}}-\frac{n}{\epsilon_{1}} t_{2} t_{3}
$$$$
t_{8}=n(n-1)+n t_{2} t_{4}
$$$$
s_{1}=\frac{N_{n+1}\left(m_{1} \eta \epsilon_{1}\right)}{M_{n+1}\left(m_{1} \eta\right)} ;
$$$$
s_{2}=\frac{N_{n}\left(m_{1} \eta \epsilon_{1}\right)}{N_{n+1}\left(m_{1} \eta\right)} ;
$$$$
s_{3}=\frac{N_{n}\left(m_{1} \eta\right)}{N_{n+1}\left(m_{1} \eta\right)}
$$

Coefficients $s_{4} \ldots s_{6}$ correspond to the coefficients $s_{1} \ldots s_{3}$ if instead of functions $N_{n}(x)$ substitute functions $J_{n}(x)$, coefficients $s_{7} \ldots s_{9}$ and $s_{10} \ldots s_{12}$ determined by the formulas for the coefficients $s_{1} \ldots s_{3}$ and $s_{4} \ldots s_{5}$ if instead of $m$ substitute $m_{2}$. The relative thickness of the aggregate $k_{s}$ and the number of waves in the circumferential direction were varied.

\section{Results and Discussions}

To solve the transcendental equation (19), the Muller method is used, and at each iteration of the Muller method, the Gauss method is used with the allocation of the main element [21]. The dispersion characteristics are understood as the dependence of the phase velocity on the wave number for different parameters of the mechanical system. This method is suitable for multilayer cylindrical bodies. The program is written in $\mathrm{C}++$.

To calculate the Bessel and Neumann functions of a complex argument of any order, the argument $z=x+i y$ was replaced by exponential functions in the form:

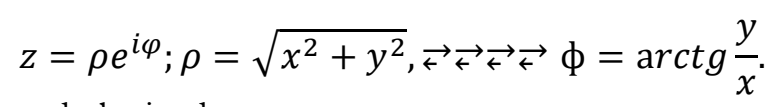

and obtained: 


$$
\begin{gathered}
J_{0}\left(\rho e^{i \varphi}\right)=\sum_{k=0}^{\infty}(-1)^{k} \frac{\left(\frac{\rho}{2}\right)^{2 k}}{(k !)^{2}} e^{2 k \varphi} \\
=U_{0}(\rho, \phi)+i V_{0}(\rho, \phi) ; \\
U_{0}(\rho, \phi)=\sum_{k=o}^{\infty}(-1)^{k} \frac{\left(\frac{\rho}{2}\right)^{2 k}}{(k !)^{2}} \cos 2 k \phi=U_{0}(\rho, \phi), \\
V_{0}(\rho, \phi)=\sum_{k=o}^{\infty}(-1)^{k} \frac{\left(\frac{\rho}{2}\right)^{2 k}}{(k !)^{2}} \sin 2 k \phi=U_{0}(\rho, \phi) .
\end{gathered}
$$

For series (20), the remainder does not exceed the first discarded term. If we choose for $\mathrm{U}_{0}(\mathrm{p}, \phi)$ and $\mathrm{V}_{0}(\mathrm{p}$, ф) 26 terms of the series (polynomials of the 50 th degree in $\rho$ ), then the error modulo will be less than the maximum value of which (for $\rho<10$ ) is approximately $1.5 \times 10-17$.

Table 1. Some values of the Bessel function depending on the argument $(\phi=100)$.

\begin{tabular}{|c|c|c|c|c|}
\hline $\mathbf{Z}$ & \multicolumn{2}{|c|}{$\mathbf{J}_{\mathbf{0}}(\mathbf{z})$} & \multicolumn{2}{c|}{$\mathbf{V}_{\mathbf{0}}(\mathbf{z})$} \\
\hline 0.0 & 0.99041 & -0.00021 & -1.97937 & 0.11159 \\
\hline 0.1 & 0.99765 & -0.00085 & -1.53476 & 0.11269 \\
\hline 0.2 & 0.99062 & -0.00340 & -1.08176 & 0.11597 \\
\hline 0.3 & 0.97895 & -0.00761 & -0.80837 & 0.11999 \\
\hline
\end{tabular}

Some values of the Bessel function depending on the argument $(\phi=100)$ are given in Table 1.

For complex roots, the Muller method simplifies calculations and provides faster convergence than the Bairstow method if the roots are close to each other [27, 28]. When using the Muller method, quadratic interpolation is applied, which leads to iterations of the form:

$$
Z^{[j+1]}=Z^{[j]]}-\left(Z^{[j]}-Z^{[j-1]}\right) \frac{2 C_{j}}{B_{j j}^{2} 4 A_{j} C_{j}} \operatorname{sign} B
$$

where

$$
\begin{gathered}
A_{j}=g_{i} f_{i}-g_{i}\left(1+g_{i}\right)^{2} f_{j-1}+g_{i} f_{i-2} ; \\
B_{j}=\left(2 g_{i}+1\right) f_{j}^{2}-\left(1+g_{i}\right)^{2} f_{j-1}+g_{i} f_{i-2} ; \\
C_{j}=\left(g_{i}+1\right) f_{i} ; f_{i}=f\left(z^{[1]}\right) ; \\
g_{i}=\frac{z^{[j}-z^{[j-1]}}{z^{[j-1]}-z^{[j-2]}} \\
j=0,1,2
\end{gathered}
$$

To start the solution, you can put $z^{[0]}=z_{00} ; z^{[1]}=$ $z_{01} ; z^{[2]}=z_{02}$.

where $z_{00}, z_{01}, z_{02}$ - are solutions of the problem in the elastic formulation.

The calculation results for relaxation kernel $R(t)=$ $A e^{-\beta t} / t^{1-\alpha}$, and for values of parameters: $A=$ 0,$048 ; \beta=0,05 ; \alpha=0,1$, are shown in Fig. 1. Figure 1 shows the dependences of the change in the phase velocity (real- $c_{R}$ and imaginary- $\left.c_{I}\right)$ from $\beta_{a}\left(\beta_{a}=\right.$ $\left.G_{0} / G_{s 0} ; G_{01}=G_{02}=G_{0}\right)$ for different values $n$ ( $\mathrm{n}=1,2, . .8$ ), when $k_{s}=10$, for $k=1, k_{s}=5$ and $k=$ $2, k_{s}=3$.

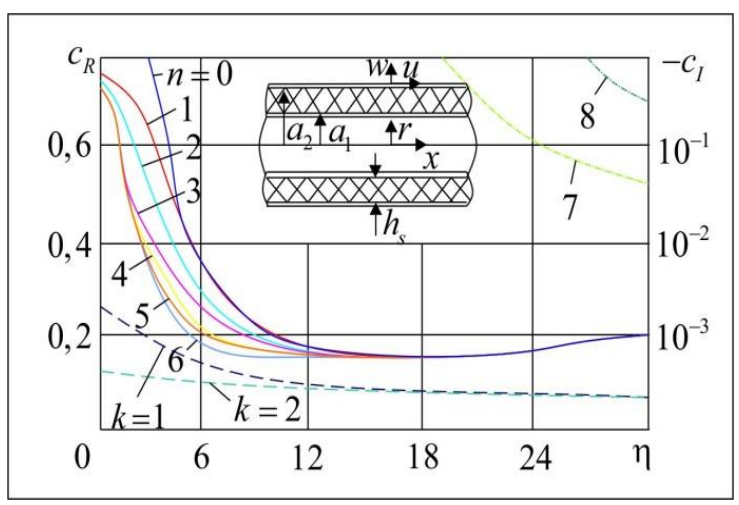

Fig. 1. Dispersion dependences for different numbers of waves in the circumferential direction (rigid contact).

The solid lines correspond to the first radial mode of motion, and the dashed lines correspond to the damping coefficient $(n=1,2 \ldots 8)$. As follows from this graph, for sufficiently long waves $\left(\beta_{a} \geq 13\right)$, the real parts of the dispersion curves of the first mode for different ones $n$ coincide. This allows us to conclude that the first resonant velocities for the problem of the effect of a moving load on a three-layer shell with axisymmetric and non-axisymmetric loads coincide. In all the considered cases of changing $n$, the variance curves coincide. The energy dissipation in the non-axisymmetric oscillation at $\left(\beta_{a} \geq 12\right)$ coincides with differences of up to $10 \%$. In the same drawing, the dashed curve refers to the special case where the inner shell is absolutely rigid. As calculations have shown, for such a shell with a rigid core, the first mode of motion does not exist, and the second (axial) mode is the lowest; moreover, the dispersion curves coincide for all of them.

The results of calculations based on equation (19) in the case of $R(t)=0$ and $\mathrm{n}=0$ are compared with the calculations obtained using the numerical method of difference schemes given in [29]. The real parts of the complex phase velocity obtained from [29] coincide with our results with a difference of up to $10 \%$. The results of the work at $R(t)=0$ and $n \neq 1$ were compared with the works $[30,31]$. The behaviour of the change in the phase velocity almost coincides with Fig. 1 (differs by up to $5 \%$ ).

Similar dependences for $k_{s}=20$ are shown in Fig. 2 . Here, the difference between symmetric and axisymmetric waves of the first mode is more significant, but with an increase in $n$ the minima of the dispersion curves do not change, but only they shifted towards longer waves. Consequently, the resonant velocities can be determined from the solution of the corresponding axisymmetric problem. For the second mode, the phase velocities at different $n$ coincide. With an increase in the 
thickness of the filler, the phase velocity of the first mode increase, the second- decrease.

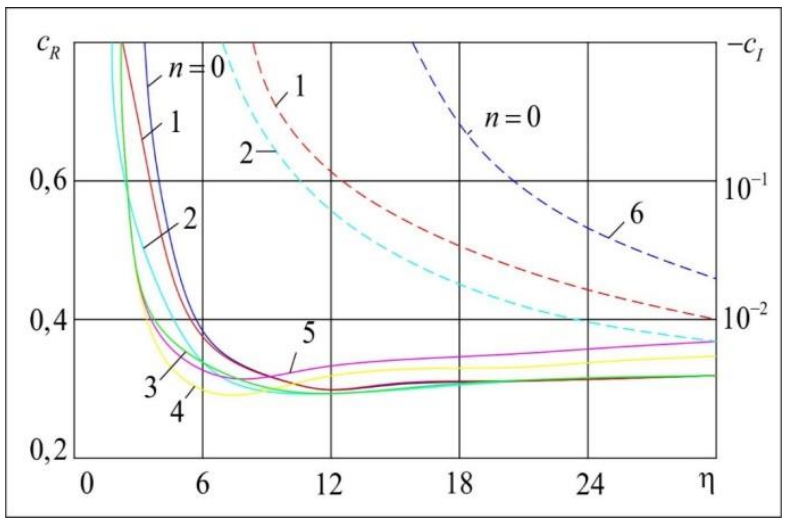

Fig. 2. Model dispersion curves (sliding contact).

Figures 3 and 4 show graphs of the change in phase velocities for a three-layered shell from $\eta$, hard and sliding contact in case of thicker filler $k_{s}=30$. Fig. 3 shows the dispersion curves of a rigid contact, and Fig. 4 shows- a sliding contact. The solid lines- is the first axial mode, the dashed lines- the damping coefficient of the axial mode of motion at different values of the number of waves in the circumferential direction.

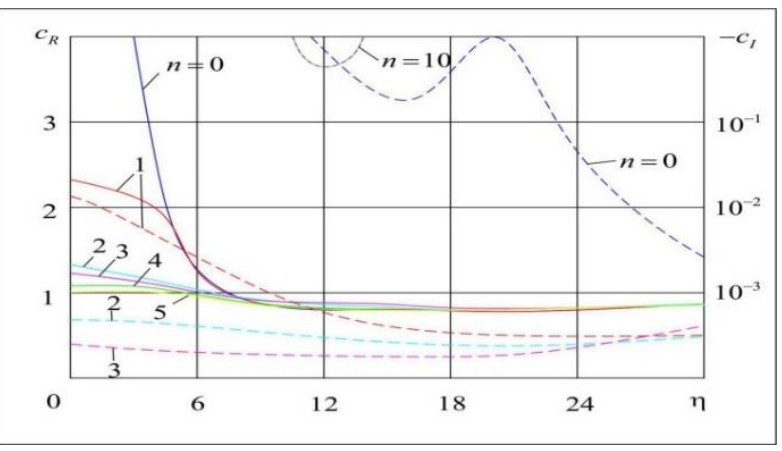

Fig. 3. Changing the phase velocity for a rigid contact.

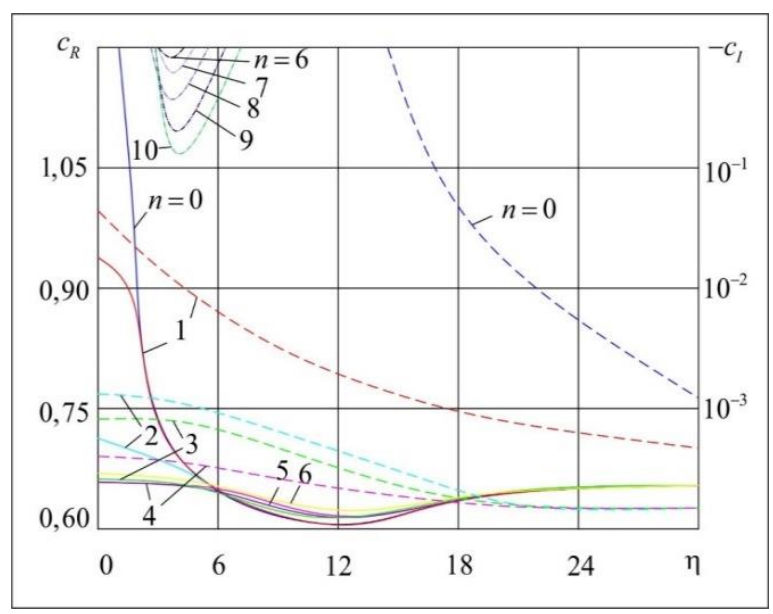

Fig. 4. Change in phase velocity for sliding contact.

As in the case of the exact solution, for $\eta \leq 12$ there is a significant difference in the dispersion curves of the first mode at different $n$; for the second mode, the curves coincide, and for the third mode, with increasing $n$ the minima decrease and they shift towards shorter waves. In determining the first (lowest) resonant velocity, one can also use the results of the axisymmetric solution.

Figure 5 shows graphs of the effect of the filler thickness $k_{S}\left(k_{s}=h_{s} / R\right)$ on the phase velocity of the first mode at different values $\eta$ for the rigid contact $(\bar{\rho}=$ $\rho_{0} / \rho_{s}, \rho_{1}=\rho_{2}=\rho_{0}, \bar{\rho}=25$ ). The picture shows 1 $\eta=25,2-\eta=50,3-\eta=75$. The solid line corresponds to the real, dashed lines - the imaginary parts of the phase velocity.

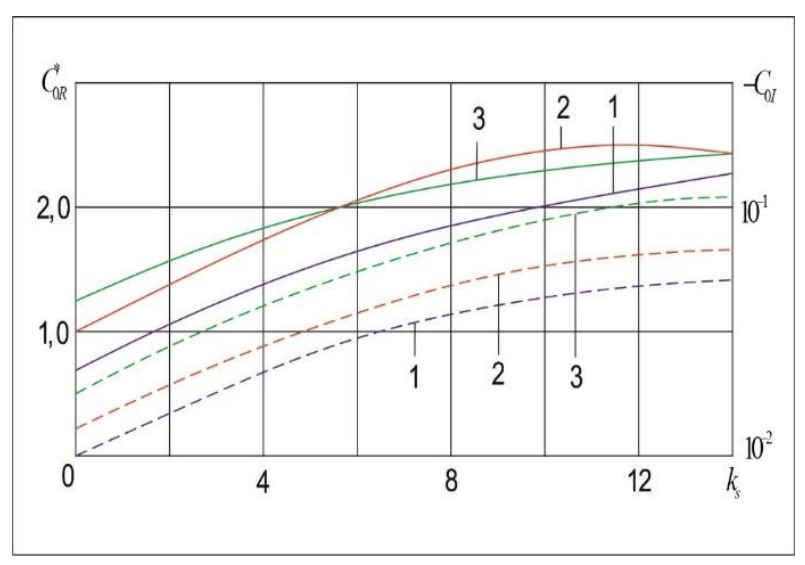

Fig. 5. Influence of the filler thickness on the phase velocity of the first mode at different wave numbers (hard contact).

It can be seen that as the filler thickness increases, the real and imaginary parts of the phase velocity gradually increase and for $k_{s} \geq 13$ approach the asymptotic.

\section{Conclusions}

A mathematical formulation of the problem of propagation of non-axisymmetric eigenwaves on a viscoelastic three-layer cylindrical body is proposed. The calculation method, algorithm and programs for calculating the dynamic behavior of a three-layer structure are developed. Formally, the dispersion equations have the same form as the corresponding dispersion equations for the elastic layer, but, unlike the latter, they are complex. Therefore, the dispersion equations in the case of a viscoelastic aggregate do not have complex conjugate roots. The absence of complexconjugate roots leads to a violation of the symmetry of the frequency spectrum of the three-layer structure.

As calculations have shown, for a shell with a rigid core, the first mode of motion does not exist, and the second (axial) mode is the lowest; moreover, the dispersion curves coincide for all of them.

In the case of short waves, there is a limit value of the complex phase velocity for all numbers of waves. The damping coefficient at the sliding contact is a nonmonotonic function of the wavenumber. 


\section{Acknowledgement}

The work was done with the financial support of the FFI of Uzbekistan (project No. F-4-14).

\section{References}

[1] T. G. Fedorova, "Experimental and theoretical study of elastic-plastic deformation, loss of stability, and supercritical behavior of cylindrical shells with loose aggregate during bending," ED "Sreda", Cheboksary, 2018.

[2] E. L. Kuznetsova, D. V. Leonenko, and E. I. Starovoitov, "Eigenvalues of three-layer circular cylindrical shells in an elastic medium," Solid State Mechanics, vol. 3, pp. 152-160, 2015.

[3] E. I. Starovoitov, Viscoelastic Layered Plates and Shells. Bel GUT, Gomel, 2002.

[4] B. H. Tan, A. D. Lucey, and R. M. Howell, "Aero/hydro-elastic stability of flexible panels: Prediction and control using localised spring support," J. Sound Vib., vol. 332, no. 26, pp. 70337054, 2013.

[5] A. Semenov, "Alexey Model of deformation stiffened orthotropic shells under dynamic loading," Journal of Siberian Federal University. Mathematics and Physics, vol. 9, no. 4, pp. 485-497, 2016.

[6] I. I. Safarov, N. R. Kulmuratov, M. K. Teshaev, and N. U. Kuldaschov, "Interaction of nonstationary waves on cylindrical body," Applied Mathematics, vol. 10, pp. 435-447, 2019. [Online]. Available: http://www.scirp.org/journal/am

[7] S. M. Bosyakov and V. Zhiwei, "Analysis of free vibrations of cylindrical fiberglass shells under Navier boundary conditions," Mechanics of Macbines, Mechanisms and Materials, vol. 3, pp. 24-27, 2011.

[8] F. S. Latifov, F. A. Seyfullaev, and S. S. Alyev, "Free vibrations reinforced by transverse ribs of an anisotropic cylindrical shell made of fiberglass with a liquid flowing in it," Applied Mechanics and Technical Physics, vol. 57, no. 4, pp. 158-162, 2016.

[9] A. I. Seyfullayev and K. A. Novruzova, "Oscillations of longitudinally reinforced orthotropic cylindrical shell filled with a viscous fluid," Eastern-European Journal of Enterprise Technologies, vol. 3, no. 7, pp. 29-33, 2015.

[10] M. Mohammadimehr, M. Moradi, and A. Loghman, "Influence of the elastic foundation on the free vibration and buckling of thin-walled piezoelectricbased FGM cylindrical shells under combined loading," J. Solid Mech., vol. 6, no. 4, pp. 347-365, 2014.

[11] P. Z. Lugovoy, "Nonstationary dynamics of inhomogeneous shell structures," Znanie, Kiev, 2005.

[12] Y. Waki, B. R. Mace, and M. J. Brennan, "Free and forced vibrations of a tyre using a wave/finite element approach," Journal of Sound and Vibration, vol. 323, pp. 737-756, 2009.
[13] P. Sabiniarz and W. Kropp, "A waveguide finite element aided analysis of the wave field on a stationary tyre, not is contact with the ground," Journal of Sound and Vibration, vol. 329, pp. 30413064, 2010.

[14] R. S. Ming, J. Pan, and M. P. Norton, "Free vibrations of elastic circular toroidal shells," Applied Acoustics, vol. 63, pp. 513-528, 2002.

[15] K. Ismayilov, "Critical stresses and deformations in a hinged-supported rectangular plate beyond the elastic limit," European Journal of Technical and Natural Sciences, vol. 3, no. 32-36, 2018.

[16] K. Ismayilov, "Critical strains and critical stresses in the steel rod beyond the Elastic limit," European Science Review, vol. 5-6, pp. 291-295, 2018.

[17] K. Ismayilov, T. Mavlonov, B. Yuldoshev, and S. Toshev, "Compressed rectangular plates stability beyond the elastic limit," CONMECHYDRO 2020. IOP Conf. Series: Materials Science and Engineering, vol. 88, p. 012199, 2020, doi:10.1088/1757899X/883/1/012199.

[18] M. A. Koltunov, A. I. Karimov, and T. Mavlyanov, "One method for solving the problem of the dynamical stability of thin-walled viscoelastic structures," Journal Mechanics of Composite Materials, vol. 16, no. 5, pp. 591-595, Sep. 1981, doi:10.1007/BF00610188.

[19] A. K. Noor and W. S. Burton, "Computational model for high temperature multilayered composite plates and shells," Applied Mech. Rev., vol. 45, no. 10, pp. 419-446, 1992.

[20] M. K. Teshaev, I. I. Safarov, N. U. Kuldashov, M. R. Ishmamatov, and T. R. Ruziev, "On the distribution of free waves on the surface of a viscoelastic cylindrical cavity, Journal of Vibrational Engineering and Technologies, vol. 8, no. 4, pp. 579-585, 2019.

[21] M. Kh. Teshaev, I. I. Safarov, and M. M. Mirsaidov, "Oscillations of multilayer viscoelastic composite toroidal pipes," J. of the Serbian Society for Computational Mechanics, vol. 13, no. 2, pp. 105-116, 2019, doi:10.24874/jsscm.2019.13.02.08

[22] H. Chao, "Multiple scattering of flexural waves in a semi-infinite thin plate with a cutout," International Journal of Solids and Structures, vol. 44, pp. 436-446, 2007.

[23] I. I. Safarov, N. U. Koldashov, and Z. I. Boltaev, "Oscillations and waves in a layered homogeneous viscoelastic medium," International Journal of New Engineering Research and Technology, vol. 6, no. 3, pp. 27-32, 2018.

[24] I. I. Safarov, N. R. Kulmuratov, and N. U. Kuldaschov, "Diffraction of surface harmonic viscoelastic waves on a multilayer cylinder with a liquid," Applied Mathematics, vol. 10, pp. 468-484, $2019 . \quad$ [Online]. Available: https://doi.org/10.1007/s42417-019-00160-x

[25] M. M. Mirsaidov, I. I. Safarov, M. K. Teshaev, and Z. I. Boltayev, "Dynamics of 
structural - Inhomogeneous coaxial-multilayered systems cylinder-shells," Journal of Physics: Conference Series, vol.1706, no. 1, p. 012033, 2020.

[26] V. V. Bolotin and Yu. N. Beginners, "Mechanics of multilayer structures," Mechanical Engineering, Moscow, 1980.

[27] M. Mirsaidov, I. Safarov, and M. Teshaev, "Dynamic instability of vibrations of thin-wall composite curvorine viscoelastic tubes under the influence of pulse pressure," E3S Web of Conferences, vol. 164, no. 14013, 2020, doi: 10.1051/e3sconf/202016414013.

[28] G. N. Watson, Theory of Bessel Functions. IL, Moscow, 1945.
[29] S. B. Filippov, N. V. Naumova, and D. N. Ivanov, "Vibrations of three-layer cylindrical shells," Bulletin of the Saint Petersburg University, Series 1: Mathematics, Mechanics, Astronomy, vol. 2, pp. 150-156, 2007.

[30] M. A. Ilgamov, V. A. Ivanov, and B. V. Gulin, "Strength, stability and dynamics of shells with elastic fillers," Nauka, Moscow, 1977.

[31] A. G. Gorshkov, "Stationary problems of dynamics of multilayer structures," Mashinostroenie, Moscow, 1992.

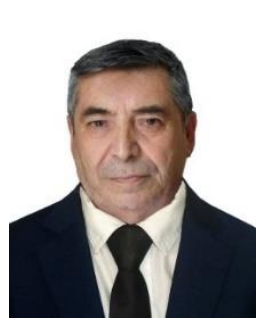

I. I. Safarov was born in the Vabkent district of Bukhara region, Republic of Uzbekistan in 1954. He received the qualification of a mechanic in the field of solid mechanics from the Tashkent National University in 1977, a Ph.D. in solid mechanics from the Moscow University of Electronic Engineering, in 1982 and a doctorate Degree in Solid Mechanics from Moscow University of Electronic Engineering, 1992From 1977 to 1991 he was a researcher at the "Foundations and Foundations" laboratory of the Institute of Mechanics and Seismic Resistance of Structures of the Academy of Sciences of the Republic of Uzbekistan. Since 1992, Professor of the Department of Mechanical Engineering of the Bukhara Technological Institute of the Food Industry, and since 2016 the head of the Department of Mathematics at the Tashkent Chemical-Technological Institute. He is the author of 24 books, over 300 articles. His research interests include processes and applications of elasticity theory, viscoelasticity theory, vibration theory, and wave propagation theory.

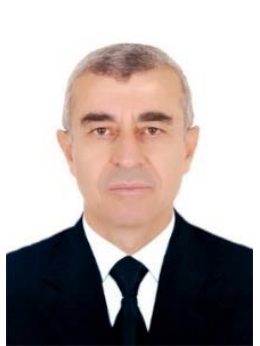

M. Kh. Teshaev was born in the Kagan district of the Bukhara region, the Republic of Uzbekistan in 1961. He received his qualification as a mechanic in theoretical mechanics from Tashkent National University in 1983, a Ph.D. in theoretical mechanics from the Kazakh National University in 1993 and a doctorate in theoretical mechanics and solid mechanics from the Institute of Mechanics and seismic resistance of structures of the Academy of Sciences of the Republic of Uzbekistan, in 2019.

From 1993 to 2019, he was a lecturer at the Department of Mathematics at the Bukhara Engineering and Technological Institute. Since 2020, the chief researcher of the Bukhara branch of the Institute of Mathematics of the Academy of Sciences of the Republic of Uzbekistan. He is the author of 11 books and over 220 articles. His research interests include processes and applications of servoconstrained systems theory, stability theory, elasticity theory, viscoelasticity theory, vibration theory.

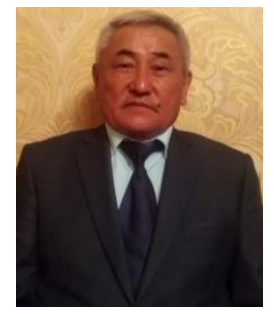

A. M. Marasulov was born in the Kibrais district of the Tashkent region, the Republic of Uzbekistan in 1956. He graduated from Tashkent State University with a degree in mathematics in 1982. The academic degree of candidate of physical and mathematical sciences in the field of theoretical mechanics was awarded by the decision of the dissertation council at the Kazakh State National University named after Al-Farabi dated December 23, 1999. The academic degree of Doctor of Technical Sciences was awarded by the decision of the Committee for Control in the Sphere of Education and Science of the Ministry of Education and Science of the Republic of Kazakhstan dated October 27, 2011.

From 1982 to 1986 he worked as a teacher of mathematics at secondary school No. 17 in the city of Chirchik, Tashkent region. From 1987 to 1990 he worked as a lecturer at the Department of Higher Mathematics at the Kazakh Chemical-Technological Institute in the city of Shymkent, Kazakhstan. From 1991 to 1993 he studied at the graduate 
school of the Moscow State University in Moscow. Since 1994, he worked as a senior lecturer, associate professor, head of the department, dean of the faculty and director of the research institute at the International Kazakh-Turkish University named after Khoja Ahmed Yasawi in the city of Turkestan. He is the author of six books, over 150 articles and four inventions. His research interests include the development of scientific foundations for assessing the dynamic strength of main pipelines, taking into account its interaction with the flow of fluid flowing in it, and the creation of methodologies for calculating strength assessment on this basis. He is a professor in the Department of Computer Science.

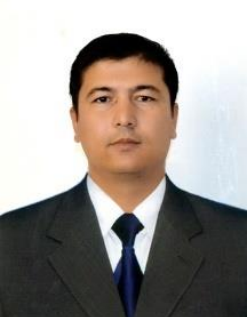

B. Z. Nuriddinov was born in Kattakurgan district of Samarkand region, Republic of Uzbekistan in 1985. He received his Bachelor's degree in Mathematics and his M.S. degree in Equations of Mathematical Physics from Bukhara State University in 2011.

From 2012 to 2019 he was an assistant at the Department of Mathematics at the Bukhara Engineering and Technological Institute. Since 2019, he has been a basic doctoral student at the Department of Mathematics of the Tashkent Institute of Chemical Technology. Published over 30 articles. He is currently working on his doctoral dissertation on the topic "Scientific foundations of the propagation of natural and non-stationary waves on three-layer cylindrical viscoelastic bodies". 